\title{
THE APPROXIMATION OF LINEAR OPERATORS
}

\author{
BY \\ J. W. BRACE( $\left.{ }^{1}\right)$ AND P. J. RICHETTA
}

\begin{abstract}
Let $L(E, F)$ be the vector space of all linear maps of $E$ into $F$. Consider a subspace $G$ of $L(E, F)$ such as all continuous maps. In $G$ distinguish a subspace $H$ of maps which are to be approximated by members of a smaller subspace $N$ of $G$. Thus we always have $N \subset H \subset G \subset L(E, F)$. Then the approximation problem which we consider is to find a locally convex linear Hausdorff topology on $G$ such that $H \subset \bar{N}, H=\bar{N}$ or the completion of $N$ is $H$.

In the case where $E$ and $F$ are Banach spaces, we have approximation topologies for (i) all linear operators, (ii) all the continuous linear operators, (iii) all weakly compact operators, (iv) all completely continuous operators, (v) all compact operators, and (vi) certain subclasses of the strictly singular operators.

Our method is that of considering members of $L(E, F)$ as linear forms on $E \otimes F^{\prime}$. Each class of linear operators is characterized as a family of linear forms. We exploit these characterizations to develop the needed topologies. Convergence on filters appears as a natural tool in doing this; indeed, in the case of linear forms we can obtain every relevant topology via convergence on filters. Particular examples give representations of weak topologies. A by-product of the main results is that Grothendieck's approximation condition holds when we have the weak topology on a locally convex space.
\end{abstract}

Let $L(E, F)$ be the vector space of all linear maps of $E$ into $F$. Consider a subspace $G$ of $L(E, F)$ such as all continuous maps. In $G$ distinguish a subspace $H$ of maps which are to be approximated by members of a smaller subspace $N$ of $G$. Thus we always have $N \subset H \subset G \subset L(E, F)$. Then the approximation problem considered here is to find a locally convex linear Hausdorff topology on $G$ such that $H \subset \bar{N}, H=\bar{N}$, or the completion of $N$ is $H$. If there is a topology such that $H \subset \bar{N}$, or $H=\bar{N}$, the same is true for every topology generating the same dual space. When the completion of $N$ is $H$, the same is true for all stronger topologies which give the same dual space of $G$. The weaker topologies are of interest in determining the size of $H$, while the stronger topologies are useful in establishing the properties of $N$ which carry over to $H$.

Members of $L(E, F)$ can be viewed as linear forms on $E \otimes F^{\prime}$. We exploit this idea in developing the topologies we need. Convergence on filters appears as a

Presented to the Society, January 26, 1969; received by the editors November 8, 1968.

AMS 1970 subject classifications. Primary 46A99, 47A63, 47B05, 47B99; Secondary 46A03, 46A20, 54A10, 54A20.

Key words and phrases. Approximation of linear operators, compact operators, weakly compact operators, completely continuous operators, tensor products, convergence on filters, completions, Grothendieck's completion theorem, Grothendieck's approximation property, weak topologies.

( ${ }^{1}$ ) The first author was partially supported by a grant from the National Science Foundation, NSF GP-7492. 
natural tool in doing this; indeed, in the case of scalar-valued functions we can obtain every relevant topology via convergence on filters [5].

Our results fall into several parts. First there are basic approximation results as found in Theorems 1.1 and 1.4. Then there are operator versus linear form descriptions of $L(E, F)$ and its subspaces. Of these we single out 2.4, 2.5 and the description in 3.3 of the weak topologies on $L(E, F)$ as representatives. Lastly, there are the approximation results stated for operators. Theorems 4.1 and 4.12 are examples in which $N$ is all continuous operators with finite-dimensional range and $H$ is taken as the linear, continuous, weakly compact, completely continuous, or compact operators. We note that Corollary 4.11 shows that Grothendieck's approximation condition holds when we have the weak topology on a locally convex space $E$.

Our notation is patterned after [9].

1. Approximation theorems. Once the linear operators are viewed as linear forms, the following approximation theorems can be utilized. The first one is an application of Grothendieck's completion theorem (see p. 248 of [9]).

1.1. THEOREM. Consider $N$ and $H$ as linear subspaces of the algebraic dual $X^{*}$ of a linear space $X$. Let $N$ separate points of $X$ and let $\mathscr{A}$ be a family of balanced, $\sigma(X, N)$-bounded subsets of $X$, covering $X$, and closed under finite sums. Then $H$ is the completion of $N$ for the topology of uniform convergence on members of $\mathscr{A}$ if and only if $H$ is all members of $X^{*}$ whose restrictions to convex hulls of members of $\mathscr{A}$ are $\sigma(X, N)$-continuous.

Proof. Since the topology of uniform convergence on $\mathscr{A}$ is the same as uniform convergence on the convex hulls of the members of $\mathscr{A}$, each linear form in the completion has restrictions to the convex hull of members of $\mathscr{A}$ which are $\sigma(X, N)$ continuous.

For the converse note that if the members of $H$ are continuous when restricted to the convex hulls of members of $\mathscr{A}$, they are uniformly continuous. This is because $\mathscr{A}$ is closed under finite sums. It follows that the restrictions to the $\sigma(X, N)$-closed convex hulls of members of $\mathscr{A}$ are continuous, and Grothendieck's completion theorem can be used to obtain the desired conclusion (see p. 248 of [9]).

The above theorem indicates that it will be necessary to characterize the operators in $H$ by the continuity of their restrictions to the members of a family $\mathscr{A}$. Trouble will occur in that we do not know if the restrictions to the convex hulls will be continuous. Thus there is need for the following corollary.

1.2. Corollary. If the linear forms in $H$ have restrictions to members of $\mathscr{A}$ which are $\sigma(X, N)$-continuous and $\mathscr{C}$ is a family of balanced convex subsets of $X$, $\mathscr{C}$ covering $X$, and such that each member of $\mathscr{C}$ is a subset of some member of $\mathscr{A}$, then $H \subset \bar{N}$ for the topology of uniform convergence on members of $\mathscr{C}$.

The proof is obtained by applying Theorem 1.1 relative to the family $\mathscr{C}$ in place of the family $\mathscr{A}$. Of course, $H$ may not have enough functions to form the completion. 
In cases where we cannot use the convex hulls of members of $\mathscr{A}$, application of the following theorem is usually more advantageous than the above corollary. It is stated first for vector valued maps. It will be used in that form to obtain the characterizations of linear operators as linear forms (see Lemma 2.1).

1.3. TheOREM. Let $E$ and $F$ be locally convex Hausdorff spaces with $E^{\prime}$ the dual of $E$. Let $F_{0}$ be a dense subspace of $F$. The linear subspace $E^{\prime} \otimes F_{0}$ of $L\left(E, F_{0}\right)$ denotes all continuous linear maps with finite-dimensional range. Let $\mathscr{A}$ denote a family of bounded, balanced subsets of $E, \mathscr{A}$ being closed under finite sums. If in $L(E, F)$ the subspace $H$ is composed of all maps whose restrictions to members of $\mathscr{A}$ are continuous, then $H$ is the closure of $E^{\prime} \otimes F_{0}$ for the topology of convergence on members of $\Phi$, where $\Phi$ is all Cauchy filters containing a member of $\mathscr{A}$. The topology is linear and locally convex on the subspace $G=\{T: T \in L(E, F)$, for each $\mathscr{F}$ in $\Phi$ and neighborhood $V$ of 0 in $F$ there is $a P$ in $\mathscr{F}$ and a positive number $n$ such that $T(P) \subset n V\}$.

Proof. From Theorem 1.3 of [3] it is seen that every map in the closure of $E^{\prime} \otimes F_{0}$ has the desired continuity. For the converse consider an arbitrary $T$ in $H$, and a neighborhood $W$ of the 0 in $L(E, F)$ for the $\Phi$-topology. It suffices to assume that $W$ is determined by a closed, convex, balanced neighborhood $V$ of 0 in $F$ and filters $\mathscr{F}_{1}, \ldots, \mathscr{F}_{n}$ in $\Phi$. (See Proposition 1 of [5] and Theorem 2.1 of [3].)

There is an $A$ in $\mathscr{A}$ such that $A \in \mathscr{F}_{i}$ for $i=1,2, \ldots, n$. The restriction of $T$ to an $A$ in $\mathscr{A}$ is uniformly continuous because $T$ is continuous on $A-A$. Let $\bar{T}$ be the continuous extension over $\bar{A}, \bar{A}$ being the closure of $A$ in the completion of $E$. Thus $\bar{T}$ defines a linear map from the linear span of $\bar{A}$ into the completion of $F$. Let $\left(x_{\imath}, \ldots, x_{n}\right)$ be the points in $\bar{A}$ to which the filters $\mathscr{F}_{1}, \ldots, \mathscr{F}_{n}$ converge respectively. There is a linearly independent set $\left(u_{1}, \ldots, u_{m}\right)$ in the linear span of $\bar{A}$ such that the points $x_{1}, \ldots, x_{n}$ are in its balanced convex hull. In $F_{0}$ there are points $z_{1}, \ldots, z_{m}$ such that $\bar{T}\left(u_{i}\right)-z_{i}$ is in $V$ for $i=1,2, \ldots, m$. Let $f$ be a continuous linear map with finite-dimensional range in $F_{0}$ and domain the completion of $E$ such that $f\left(u_{i}\right)=z_{i}$ for $i=1, \ldots, m$. Thus,

$$
\bar{T}\left(x_{i}\right)-f\left(x_{i}\right)=\sum_{j=1}^{m} a_{j}\left(\bar{T}\left(u_{j}\right)-f\left(u_{j}\right)\right)=\sum_{j=1}^{m} a_{j}\left(\bar{T}\left(u_{j}\right)-z_{j}\right)
$$

is in $V$ where $\left\{a_{j}: j=1, \ldots, m\right\}$ is a set of scalars such that

$$
\sum_{j=1}^{m}\left|a_{j}\right| \leqq 1 \text { and } x_{i}=\sum_{j=1}^{m} a_{j} u_{j}
$$

This can be done for each $x_{i}$.

Since $f$ is continuous on the completion of $E, \bar{T}$ is continuous on $\bar{A}$, and $\bar{T}\left(x_{i}\right)$ $-f\left(x_{i}\right) \in V$, it follows that there is a set $P_{i}$ in $\mathscr{F}_{i}$ such that $T(x)-f(x)$ is in $V$ for all $x$ in $P_{i}$, for $i=1, \ldots, n$. In other words, $T-f$ is in the $\Phi$-neighborhood $W$. Thus the closure of $E^{\prime} \otimes F_{0}$ is $H$. 
The space $G$ defined in the theorem is the largest subspace of $L(E, F)$ on which the $\Phi$-topology is linear (see Theorem 2.2 of [3]).

The next theorem is a variation of the one above, but for scalar valued functions. It will be used in $\S 4$. It is not a corollary but the proof is analogous.

1.4. TheOREm. Consider a subspace $N$ of $X^{*}$ where $N$ separates points of $X$. Let $\mathscr{A}$ be a family of balanced subsets of $X, \mathscr{A}$ being closed under finite sums. If $H$ is the subspace of $X^{*}$ composed of all maps whose restrictions to members of $\mathscr{A}$ are $\sigma(X, N)$-continuous then $\bar{N}=H$ for the topology on $X^{*}$ of convergence on member of $\Phi, \Phi$ being all $\sigma(X, N)$ Cauchy filters which contain at least one member of $\mathscr{A}$. The topology is a locally convex linear topology when restricted to the subspace $G=\left\{f: f \in X^{*}, f\right.$ is bounded on each member $\mathscr{F}$ of $\left.\Phi\right\}$, and $H \subset G$. (A map $f$ is bounded on a filter $\mathscr{F}$ if there is a $P$ in $\mathscr{F}$ such that $f(P)$ is bounded [5].)

The $\Phi$-topology of Theorem 1.4 always gives a $\sigma\left(G, G^{\prime}\right)$-topology on $G$ (see Proposition 4 of [5]). In $\S 3$ it is necessary to develop stronger topologies with the same dual space. Without the restriction to a given dual space, we have no constructive method for finding the largest topologies. The Hausdorff Maximal Principle does give their existence. This is stated in the following proposition.

1.5. Proposition. If there is a locally convex linear Hausdorff topology on a linear space $G$ such that for the subspaces $N$ and $H, H \subset \bar{N}, H=\bar{N}$, or the completion of $N$ is $H$, then there is at least one maximal topology with the same property.

2. Linear forms. We now characterize several classes of linear operators as linear forms. The vector space $L(E, F)$ of all linear maps of a vector space $E$ into a vector space $F$ is a collection of bilinear forms defined on $E \times F^{\#}, F^{\#}$ being a subspace of $F^{*}$ which separates points of $F$. The bilinear forms are made into linear forms by replacing $E \times F^{\#}$ by the tensor product $E \otimes F^{\#}$ (see p. 366 of [9]). We will speak of members of $L(E, F)$ as linear forms without mention of the injection.

The following lemma is a fundamental building block for our characterizations.

2.1. Lemma. Let $E$ and $F$ be linear spaces and let $E^{\prime}$ and $F^{\#}$ be subspaces of $E^{*}$ and $F^{*}$ respectively. Both subspaces separate points of the respective $E$ and $F$. In $F^{\#}$ let $\mathscr{B}$ denote a family of balanced, convex and $\sigma\left(F^{\#}, F\right)$-bounded subsets. Let $\mathscr{A}$ denote a family of balanced, $\sigma\left(E, E^{\prime}\right)$-bounded subsets of $E$. Both $\mathscr{A}$ and $\mathscr{B}$ are closed under finite sums and cover $E$ and $F^{\#}$ respectively. The following are equivalent:

(i) The linear operator $T$ is defined on $E$ with range in $\hat{F}$ such that the restrictions of $T$ to members of $\mathscr{A}$ are $\sigma\left(E, E^{\prime}\right)$-continuous. (The space $\hat{F}$ is the linear topological space formed by completing $F$ in the topology of uniform convergence on members of $\mathscr{B}$.)

(ii) The linear operator $T$ is defined on $E$ with range in $\hat{F}$ such that $T(A)$ is precompact for each $A$ in $\mathscr{A}$ and the restrictions of $T$ to members of $\mathscr{A}$ are $\sigma\left(E, E^{\prime}\right)$ continuous for the $\sigma\left(\hat{F}, F^{\#}\right)$-topology on $\hat{F}$. 
(iii) The linear form $T$ defined on $E \otimes F^{\#}$ has a restriction to $A \otimes B$ which is $\sigma\left(E \otimes F^{\#}, E^{\prime} \otimes F\right)$-continuous for each $A$ in $\mathscr{A}$ and $B$ in $\mathscr{B} .(A \otimes B$ denotes the set $\{a \otimes b: a \in A, b \in B\}$.)

(iv) The linear form $T$ defined on $E \otimes F^{\#}$ has an extension $\bar{T}$ defined on the linear span of $\bigcup\{\bar{A} \otimes \bar{B}: A \in \mathscr{A}, B \in \mathscr{B}\}$ in $E^{\prime \prime} \otimes F^{\prime}$ such that the restriction of $\bar{T}$ to $\bar{A} \otimes \bar{B}$ is $\sigma\left(E^{\prime \prime} \otimes F^{\prime}, E^{\prime} \otimes F\right)$-continuous for each $A$ in $\mathscr{A}$ and $B$ in $\mathscr{B}$. (The space $\hat{F}^{\prime}=F^{\prime}$ is the dual of $\hat{F}$ and $E^{\prime \prime}$ is the bidual of $E$ for the $\sigma\left(E, E^{\prime}\right)$-topology. The sets $\bar{A}$ and $\bar{B}$ are the closures of $A$ and $B$ in $E^{\prime \prime}$ and $\hat{F}^{\prime}$ for the $\sigma\left(E^{\prime \prime}, E^{\prime}\right)$ and $\sigma\left(\hat{F}^{\prime}, F\right)$ topologies respectively.)

Proof. First we will show that (i) implies (iv). There is no harm in considering $\hat{F}$ as having the topology of uniform convergence on members of $\{\bar{B}: B \in \mathscr{B}\}$. Since $\mathscr{A}$ is closed under finite sums, the restrictions of the linear operator $T$ to members of $\mathscr{A}$ are uniformly continuous. It can be verified that the continuous extensions over members of $\{\bar{A}: A \in \mathscr{A}\}$ piece together to form a linear map $\bar{T}$ from the linear span of $\bigcup\{\bar{A}: A \in \mathscr{A}\}$ into $\hat{F}$. This defines a linear form on the linear span of $\bigcup\{\bar{A} \otimes \bar{B}: A \in \mathscr{A}, B \in \mathscr{B}\}$.

We apply Theorem 1.3 to the linear map $\bar{T}$. Thus there is a filter $\mathscr{G}$ in $E^{\prime} \otimes F$ converging to $\bar{T}$ for the topology of convergence on all Cauchy filters containing an $\bar{A}, A$ ranging through $\mathscr{A}$. For each $\bar{A}$ is $\sigma\left(E^{\prime \prime}, E^{\prime}\right)$-compact, thus each ultrafilter containing $\bar{A}$ is a Cauchy filter. We now have that $\mathscr{G}$ converges to $\bar{T}$ on every ultrafilter containing $\bar{A}$. This is equivalent to almost uniform convergence on $\bar{A}$ (see Corollary 2.4 of [3]). From Definition 2.1 of [1] for quasi-uniform convergence and Definition 2.3 of [1] for almost uniform convergence, we are able to express almost uniform convergence on $\bar{A}$ in the following manner. For an arbitrary positive number $\varepsilon$, a $\bar{B}$, and a set $G$ from a refinement of $\mathscr{G}$, there is a finite subset $\left(g_{1}, \ldots, g_{n}\right)$ of $G$ such that

$$
\inf _{i} \sup _{y^{\prime} \in \bar{B}}\left|\left\langle g_{i}(x), y^{\prime}\right\rangle-\left\langle\bar{T}(x), y^{\prime}\right\rangle\right|<\varepsilon
$$

for all $x$ in $\bar{A}$.

Now consider the maps as linear forms. Along with pointwise convergence on $\bar{A} \otimes \bar{B}$ the above statement implies that $\inf _{i}\left|g_{i}\left(x \otimes y^{\prime}\right)-\bar{T}\left(x \otimes y^{\prime}\right)\right|<\varepsilon$ for all $x \otimes y^{\prime}$ in $\bar{A} \otimes \bar{B}$. Thus, when considered as linear forms, the filter $\mathscr{G}$ converges almost uniformly to $\bar{T}$ on $\bar{A} \otimes \bar{B}$. The restriction of the linear form $\bar{T}$ to $\bar{A} \otimes \bar{B}$ is $\sigma\left(E^{\prime \prime} \otimes \hat{F}^{\prime}, E^{\prime} \otimes F\right)$-continuous because almost uniform convergence preserves continuity (see Theorem 2.2 of [1]).

To verify that (iv) implies (i), consider an arbitrary $x$ in $E$ and observe that $\bar{T}(x)=T(x)$ is a linear form defined on $F^{\prime}$ and its restriction of each $\bar{B}$ is $\sigma\left(F^{\prime}, F\right)$ continuous. The Grothendieck completion theorem (p. 248 of [9]) says that $T(x)$ is in $\hat{F}$. In order to prove the desired continuity, assume that there is an $\bar{A}$ on which the restriction of $\bar{T}$ is not continuous. Thus there is an $x_{0}$ in $\bar{A}$ and a filter $\mathscr{F}$ containing $\bar{A}$ and converging to $x_{0}$ such that $\bar{T}(\mathscr{F})$ does not converge to $\bar{T}\left(x_{0}\right)$ 
in $\hat{F}$. In other words, there is a positive number $\varepsilon$, a $\bar{B}$ and a set $C$ from a refinement of $\mathscr{F}$ such that $\sup _{y^{\prime} \in \bar{B}}\left|\left\langle\bar{T}(x), y^{\prime}\right\rangle-\left\langle\bar{T}\left(x_{0}\right), y^{\prime}\right\rangle\right|>\varepsilon$ for all $x$ in $C$.

For each set $D$ from the refinement of $\mathscr{F}$ which contains $C$, let

$$
U_{D}=\left\{x \otimes y^{\prime}:\left|\left\langle\bar{T}(x), y^{\prime}\right\rangle-\left\langle\bar{T}\left(x_{0}\right), y^{\prime}\right\rangle\right|>\varepsilon \text { for } x \text { in } D \text { and } y^{\prime} \text { in } \bar{B}\right\} .
$$

These sets form the basis of a filter $\mathscr{F}$. Let $\mathscr{U}$ be an ultrafilter which is a refinement of $\bar{F}$. The ultrafilter $\mathscr{U}$ converges to the point $x_{0} \otimes y_{0}^{\prime}$ in $\bar{A} \otimes \bar{B}$ for the $\sigma\left(E^{\prime \prime} \otimes \hat{F}^{\prime}, E^{\prime} \otimes F\right)$-topology. The point $x_{0}$ is still the limit of the filter $\mathscr{F}$ and $y_{0}$ is an appropriate member of $\bar{B}$. This is because of the compactness of $\bar{A} \otimes \bar{B}$. As constructed, $\bar{T}(\mathscr{U})$ does not converge to $\bar{T}\left(x_{0} \otimes y_{0}^{\prime}\right)$. This contradicts the continuity of the linear form $\bar{T}$ and gives the desired result.

To go from (i) to (ii) observe that $T$ is uniformly continuous when restricted to each member $A$ of $\mathscr{A}$ because the restriction to $A-A$ is continuous. Thus $T$ maps precompact sets onto precompact sets. The weakening of the topology on the range does not disturb continuity. A straightforward attack will verify the converse.

The equivalence of (iv) and (iii) is seen by restricting $\bar{T}$ to obtain $T$, and by extending $T$ to obtain $\bar{T}$.

2.2. Remark. In statement (iii) of Lemma 2.1 the roles of $E, E^{\prime}$ and $\mathscr{A}$ may be interchanged with $F^{\#}, F$ and $\mathscr{B}$ without any actual change in the statement. Such a change in (i) and (iii) would concern a dual operator $T^{\prime}$ defined on $F^{\#}$. It is the transpose of a linear operator $T$ defined on $E$.

The following statement is equivalent to the statements of Lemma 2.1 when the members of $\mathscr{A}$ are convex.

(i') The linear operator $T^{\prime}$ is defined on $F^{\#}$ with range in the completion of $E^{\prime}$ for the topology of uniform convergence on members of $\mathscr{A}$, and the restrictions of $T^{\prime}$ to members of $\mathscr{B}$ are continuous for the $\sigma\left(F^{\#}, F\right)$-topology.

2.3. Remark. The advantage of statement (iv) of Lemma 2.1 over statement (iii) is that $\bar{A} \otimes \bar{B}$ is a compact subset of $E^{\prime \prime} \otimes \hat{F}^{\prime}$ for the $\sigma\left(E^{\prime \prime} \otimes \hat{F}^{\prime}, E^{\prime} \otimes F\right)$-topology. This makes it possible to conclude that the restriction of $\bar{T}$ to finite sums of such sets will also be continuous. The linear form $T$ will thus be continuous when restricted to sets of the form $\left(A_{1} \otimes B_{1}\right)+\left(A_{2} \otimes B_{2}\right)+\cdots+\left(A_{n} \otimes B_{n}\right)$. All finite sums of members of $\{A \otimes B: A \in \mathscr{A}, B \in \mathscr{B}\}$ are denoted by $\mathscr{A} \otimes \mathscr{B}$, and the restrictions of $T$ to members of $\mathscr{A} \otimes \mathscr{B}$ are thus continuous.

Many different classes of sets can be used for the families $\mathscr{A}$ and $\mathscr{B}$. When $E$ and $F$ are normed spaces we will use the following notation for such families. For the family $\mathscr{A}$ the following collections are used:

$S=($ all scalar multiples of the unit ball of $E)$.

$S^{\prime \prime}=$ (all scalar multiples of the unit ball of $E^{\prime \prime}$ ).

$J=($ all convex balanced hulls of finite subsets of $E)$.

$J^{\prime \prime}=$ (all convex balanced hulls of finite subsets of $E^{\prime \prime}$ ).

$K^{0}=$ (all precompact subsets of $E$ for the norm topology).

$K^{\prime}=$ (all subsets of $E$ which are precompact for the topology of uniform convergence on convex $\sigma\left(E^{\prime}, E^{\prime \prime}\right)$-compact subsets of $\left.E^{\prime}\right)$. 
$K^{2}=\left(\right.$ all $\sigma\left(E, E^{\prime}\right)$-compact subsets of $\left.E\right)$.

The following families will be used for $\mathscr{B}$ :

$S^{\prime}=\left(\right.$ all scalar multiples of the unit ball of $F^{\prime}$ ).

$J^{\prime}=$ (all balanced convex hulls of finite subsets of $F^{\prime}$ ).

It is important to note that the families $S \otimes J^{\prime}, S^{\prime \prime} \otimes J^{\prime}$, and $J \otimes S^{\prime}$ contain the convex hulls of every set which they contain.

2.4. THEOREM. Let $E$ and $F$ be normed linear spaces and $\hat{F}$ the completion of $F$. On $E \otimes \hat{F}^{\prime}$ and $E^{\prime \prime} \otimes \hat{F}^{\prime}$ place the $\sigma\left(E^{\prime \prime} \otimes \hat{F}^{\prime}, E^{\prime} \otimes F\right)$-topology.

(i) The linear operators which map $E$ into $\hat{F}$ are all the linear forms on $E \otimes \hat{F}^{\prime}$ whose restrictions to members of $J \otimes S^{\prime}$ are continuous.

(ii) The continuous linear operators mapping $E$ into $\hat{F}$ are all the linear forms whose restrictions to any one of the following families are continuous:

(a) $\left(J \otimes S^{\prime}\right)+\left(S \otimes J^{\prime}\right)$,

(b) $K^{0} \otimes S^{\prime}$,

(c) $\left(K^{0} \otimes S^{\prime}\right)+\left(S \otimes J^{\prime}\right)$.

(iii) The weakly compact linear operators (continuous linear operators which map bounded sets into weakly compact subsets of $\hat{F}$ ) are all the linear forms defined on $E^{\prime \prime} \otimes F^{\prime}$ whose restrictions to any one of the following families are continuous:

(a) $\left(J^{\prime \prime} \otimes S^{\prime}\right)+\left(S \otimes J^{\prime}\right)$,

(b) $\left(J^{\prime \prime} \otimes S^{\prime}\right)+\left(K^{\prime} \otimes S^{\prime}\right)$.

(iv) The completely continuous operators (continuous linear operators which map weakly compact subsets of $E$ into compact subsets of $\hat{F}$, see [8]) are all the linear forms on $E \otimes \hat{F}^{\prime}$ whose restrictions to one of the following families are continuous:

(a) $K^{2} \otimes S^{\prime}$,

(b) $\left(K^{2} \otimes S^{\prime}\right)+\left(S \otimes J^{\prime}\right)$,

(v) The compact operators (continuous linear operators which map bounded subsets of $E$ into compact subsets of $\hat{F}$ ) are all the linear forms on $E \otimes F^{\prime}$ whose restrictions to members of $S \otimes S^{\prime}$ are continuous.

(vi) The operators which form the closure of $E^{\prime} \otimes F$ for norm topology are all linear forms whose restrictions to convex hulls of members of $S \otimes S^{\prime}$ are continuous. (The norm topology for $L(E, \hat{F})$ is uniform convergence on members of $S$.)

Proof. (i) A linear operator maps $E$ into $\hat{F}$ if and only if its restrictions to members of $J$ are $\sigma\left(E, E^{\prime}\right)$-continuous. Apply Lemma 2.1 (i) and (iii).

(ii) A linear operator defined on the normed space $E$ is continuous if and only if its restrictions to members of $S$ are $\sigma\left(E, E^{\prime}\right)$-continuous for the $\sigma\left(\hat{F}, \hat{F}^{\prime}\right)$-topology on $\hat{F}$. Lemma 2.1 says that the corresponding linear forms have continuous restrictions on members of $S \otimes J^{\prime}$, but such linear forms may not correspond to linear operators which map into $\hat{F}$. To keep the range in the correct space we make the additional requirement that the linear forms have continuous restrictions to members of $J \otimes S^{\prime}$. Use part (iv) of Lemma 2.1 to extend the linear forms over $E^{\prime \prime} \otimes F^{\prime}$ with continuous restrictions to members of $S^{\prime \prime} \otimes J^{\prime}$. The restrictions to 
members of $J \otimes S^{\prime}$ remain continuous. Because of the compactness of the sets involved, the restrictions to members of $\left(J \otimes S^{\prime}\right)+\left(S^{\prime \prime} \otimes J^{\prime}\right)$ are continuous. Thus the restrictions to members of $\left(J \otimes S^{\prime}\right)+\left(S \otimes J^{\prime}\right)$ are continuous and such linear forms represent the continuous maps.

For part (b) observe that the continuous operators are precisely the operators whose restrictions to members of $K^{0}$ are continuous for the norm topologies on both range and domain. On members of $K^{0}$ the norm and $\sigma\left(E, E^{\prime}\right)$-topologies are identical. From Lemma 2.1 we see that our operators are all linear forms with continuous restrictions to members of $K^{0} \otimes S^{\prime}$. In this case there is no problem about the range because $J \otimes S^{\prime}$ is a subset of $K^{0} \otimes S^{\prime}$.

To obtain part (c) extend the linear forms over $E^{\prime \prime} \otimes \hat{F}^{\prime}$ by application of (iv) of Lemma 2.1 relative to $S \otimes J^{\prime}$. The members of $K^{0} \otimes J^{\prime}$ and $S \otimes J^{\prime}$ can now be replaced by compact sets. Thus the linear forms will have continuous restrictions to members of $\left(K^{0} \otimes J^{\prime}\right)+\left(S \otimes J^{\prime}\right)$.

(iii) The weakly compact operators are continuous. Thus the corresponding linear forms must have continuous restrictions on members of $S \otimes J^{\prime}$. By Lemma 2.1 there is a continuous extension over $E^{\prime \prime} \otimes F^{\prime}$ with continuous restrictions to members of $S^{\prime \prime} \otimes J^{\prime}$. The corresponding operators are weakly compact if and only if $E^{\prime \prime}$ is mapped into $\hat{F}$ (see p. 482, Theorem 2 of [6]). This is equivalent to requiring that the restrictions of the linear forms to members of $J^{\prime \prime} \otimes S^{\prime}$ are continuous. Since the members of $J^{\prime \prime} \otimes S^{\prime}$ and $S^{\prime \prime} \otimes J^{\prime}$ are compact, the linear forms will have continuous restrictions to members of $\left(J^{\prime \prime} \otimes S^{\prime}\right)+\left(S \otimes J^{\prime}\right)$.

Because the adjoint of a weakly compact operator is weakly compact, a weakly compact operator is continuous for the topology on $E$ of uniform convergence on the $\sigma\left(E^{\prime}, E^{\prime \prime}\right)$-compact convex subsets of $E^{\prime}$ into $\hat{F}$ with the norm topology. Thus a weakly compact operator takes the members of $K^{\prime}$ into norm compact subsets of $F$. We apply Lemma 2.1 to see that the resulting linear form has continuous restrictions to members of $K^{\prime} \otimes S^{\prime}$. Every such linear form corresponds to a continuous linear operator because $K^{0} \otimes S^{\prime} \subset K^{\prime} \otimes S^{\prime}$. Because the members of $K^{\prime}$ can be replaced by their $\sigma\left(E^{\prime \prime}, E^{\prime}\right)$-compact closures in $E^{\prime \prime}$, we are able to say that the restrictions to members of $\left(J^{\prime \prime} \otimes S^{\prime}\right)+\left(K^{\prime} \otimes S^{\prime}\right)$ are continuous. This completes (iii(b)).

Parts (iv) and (v) are proved by the same methods.

(vi) Theorem 1.1 gives the desired result because the norm topology on the linear operators becomes the topology of uniform convergence on members of $S \otimes S^{\prime}$ when the operators are viewed as linear forms.

The above theorem does not exhaust the possibilities as the following corollary shows.

2.5. Corollary. A linear form on $E^{\prime \prime} \otimes F^{\prime}$ whose restrictions to members of $\left(J^{\prime \prime} \otimes S^{\prime}\right)+\left(K^{2} \otimes S^{\prime}\right)+\left(S \otimes J^{\prime}\right)$ are $\sigma\left(E^{\prime \prime} \otimes \hat{F}^{\prime}, E^{\prime} \otimes F\right)$-continuous corresponds to a strictly singular operator which maps the normed space $E$ into the Banach space $\hat{F}$. 
Proof. The definition of a strictly singular operator can be found in [8] where it is shown in Corollary 5 that an operator which is weakly compact and completely continuous is strictly singular. Parts (iii) and (iv) of the theorem show that the linear form corresponds to such an operator.

3. Topologies. When the linear operators in $L(E, F)$ are considered as linear forms on $E \otimes F^{\#}$ it is known that every locally convex linear topology can be represented as convergence on a family of filters composed of subsets of $E \otimes F^{\#}$ [5]. It is not clear as to how these topologies are to be represented if it is mandatory that one consider the linear operators and not the linear forms.

From an operator point of view, a general way of topologizing $L(E, F)$ is by convergence on a family $\Phi$ of filters composed of subsets of $E$ with some kind of a topology on $F$. Every locally convex topology on $F$ can be obtained by convergence on a family $\Psi$ of filters composed of subsets of $F^{\#}, F^{\#}$ being a subspace of $F^{*}$ which separates points of $F$. Thus, many of the topologies on $L(E, F)$ can be characterized by means of the two families $\Phi$ and $\Psi$. The next step is to determine what such topologies will be in terms of convergence on filters composed of subsets of $E \otimes F^{\#}$.

3.1. Definition. (i) The family $\Phi \otimes \Psi$ is composed of filters of the form $\mathscr{F} \otimes \mathscr{G}$ for all $\mathscr{F}$ in $\Phi$ and all $\mathscr{G}$ in $\Psi$. The filter $\mathscr{F} \otimes \mathscr{G}$ is composed of subsets of $E \otimes F^{\#}$ with the family $(A \otimes B: A \in \mathscr{F}, B \in \mathscr{G})$ as a basis.

(ii) The family $\Phi \mid \Psi$ is composed of all filters of the form $\mathscr{F} \mid \mathscr{G}$ for $\mathscr{F}$ in $\Phi$ and $\mathscr{G}$ in $\Psi$. The filter $\mathscr{F} \mid \mathscr{G}$ has the family $\left\{\bigcup_{x \in A}\{x\} \otimes \alpha(x): A\right.$ in $\mathscr{F}$ and for every map $\alpha$ of $A$ into $\mathscr{G}\}$ as a basis.

(iii) The family $\Psi \mid \Phi$ is defined by reversing the roles of $\Phi$ and $\Psi$ in part (ii).

3.2. Proposition. (i) The families $\Phi \mid \Psi$ and $\Psi \mid \Phi$ are composed of refinements of members of $\Phi \otimes \Psi$ and thus on $\left(E \otimes F^{\#}\right)^{*}$ the topology of convergence on members of $\Phi \otimes \Psi$ is stronger than either the $\Phi \mid \Psi$-topology or the $\Psi \mid \Phi$-topology.

(ii) The family $\Phi \otimes \Psi$ is the family $\Phi \mid \Psi$ and the resulting topologies are the same if and only if, for every $\mathscr{F}$ in $\Phi, \mathscr{G}$ in $\Psi, A$ in $\mathscr{F}$, and mapping $\alpha$ of $A$ into $\mathscr{G}$, there exists an $A_{0}$ in $\mathscr{F}$ such that $A_{0} \subset A$ and $\bigcap\left\{\alpha(x): x\right.$ in $\left.A_{0}\right\}$ is in $\mathscr{G}$.

(iii) If $\cap\{B: B$ in $\mathscr{G}\}$ is in $\mathscr{G}$ for all $\mathscr{G}$ in $\Psi$ then $\Phi \otimes \Psi=\Phi \mid \Psi$.

(iv) If the set $\bigcap\{A: A$ in $\mathscr{F}\}$ is of finite cardinality and a member of $\mathscr{F}$ for every $\mathscr{F}$ in $\Phi$, then $\Phi \otimes \Psi=\Phi|\Psi=\Psi| \Phi$. (The same conclusion is true with $\Phi$ replacing $\Psi$ in the hypothesis.)

(v) If $F$ is given the $\Psi$-topology and the linear subspace $G$ of $L(E, F)$ is given the topology of convergence on members of $\Phi$, then when $G$ is considered as linear forms on $E \otimes F^{\#}$ the topology is convergence on members of $\Phi \mid \Psi$.

(vi) If a linear space $G$ of linear forms defined on $E \otimes F^{\#}$ is given the topology of convergence on members of $\Psi \backslash \Phi$, then when $G$ is considered as a subspace of $L\left(F^{\#}, E^{*}\right)$ the topology is convergence on members of $\Psi$ with $E^{*}$ having the topology of convergence on members of $\Phi$. 
(vii) (Uniform Convergence.) Let $\mathscr{A}$ be a family of subsets of $E, \Phi$ being the family of filters having a single member of $\mathscr{A}$ as a basis, and $\mathscr{B}$ a family of subsets of $F^{\#}, \Psi$ being the family of filters having a single member of $\mathscr{B}$ as a basis. The topology on the linear operators of uniform convergence on members of $\mathscr{A}$ with $F$ having the topology of uniform convergence on members of $\mathscr{B}$ is the same as the topology on the linear forms of convergence on members of $\Phi \otimes \Psi=\Phi|\Psi=\Psi| \Phi$.

Proof. (i) Consider an arbitrary $A \otimes B$ from an arbitrary $\mathscr{F} \otimes \mathscr{G}$ in $\Phi \otimes \Psi$. Let $\alpha$ be a map from $A$ into $\mathscr{G}$ such that $\alpha(x)=B$ for all $x$ in $A$. Note that $\bigcup\{(x) \otimes \alpha(x): x \in A\}=\bigcup\{(x) \otimes B: x \in A\}=A \otimes B$. Thus $A \otimes B$ is in $\mathscr{F} \mid \mathscr{G}$ and $\mathscr{F} \backslash \mathscr{G}$ is a refinement of $\mathscr{F} \otimes \mathscr{G}$. In the same manner $\mathscr{G} \mid \mathscr{F}$ is also a refinement of $\mathscr{F} \otimes \mathscr{G}$.

(ii) Assume that $\mathscr{F} \otimes \mathscr{G}=\mathscr{F} \mid \mathscr{G}$. Thus, given $A$ in $\mathscr{F}$ and a map $\alpha$ of $A$ into $\mathscr{G}$, there exists an $A_{0}$ in $\mathscr{F}$ and a $B_{0}$ in $\mathscr{G}$ such that $A_{0} \otimes B_{0} \subset \bigcup_{x \in A}(x) \otimes \alpha(x)$. In other words, for each $x_{0}$ in $A_{0}, x_{0} \otimes B_{0} \subset \bigcup_{x \in A}(x) \otimes \alpha(x)$. This implies that $A_{0} \subset A$ and $B_{0} \subset \alpha(x)$ for all $x$ in $A_{0}$ giving the result that $\bigcap\left\{\alpha(x): x \in A_{0}\right\}$ is in $G$.

For the converse, consider the set $\bigcup_{x \in A}(x) \otimes \alpha(x)$ from $\mathscr{F} \backslash \mathscr{G}$. It is assumed that there is an $A_{0}$ in $\mathscr{F}$ such that $A_{0} \subset A$ and $B_{0}=\bigcap\left\{\alpha(x): x \in A_{0}\right\}$ is in $\mathscr{G}$. Thus $A_{0} \otimes B_{0} \subset \bigcup_{x \in A}(x) \otimes \alpha(x)$ and $\mathscr{F} \otimes \mathscr{G}$ is a refinement of $\mathscr{F} \backslash \mathscr{G}$. Combine this with part (i) to see that $\mathscr{F} \otimes \mathscr{G}=\mathscr{F} \mid \mathscr{G}$.

(iii) Since $\bigcap\{B: B \in \mathscr{G}\} \subset \bigcap\{\alpha(x): x \in A\}$ for every map $\alpha$ and $A$ in an $\mathscr{F}$ from $\Phi$, part (ii) can be applied to obtain the conclusion.

(iv) We can use (iii) with $\Phi$ and $\Psi$ interchanged to see that $\Phi \otimes \Psi=\Psi \mid \Phi$. Continue by considering an arbitrary $A$ from a member $\mathscr{F}$ of $\Phi$ and a mapping $\alpha$ of $A$ into $\mathscr{G}$. The hypothesis says that there is an $A_{0}$ in $\mathscr{F}$ such that $A_{0}$ is of finite cardinality and $A_{0} \subset A$. Thus, $\bigcap\left\{\alpha(x): x \in A_{0}\right\}$ is in $\mathscr{G}$ and (ii) can be applied to obtain the desired conclusion.

(v) In order to obtain a member of a neighborhood subbase for the topology on $F$ of convergence on members of $\Psi$, consider a positive number $\varepsilon$ and a member $\mathscr{G}$ of $\Psi$. The desired set is $U(\varepsilon, \mathscr{G})=\left\{y\right.$ : there is a $B$ in $\mathscr{G}$ such that $\left|\left\langle y, y^{\prime}\right\rangle\right| \leqq \varepsilon$ for all $y^{\prime}$ in $B$ \} [3]. A subbase of the neighborhoods of 0 in $G$ for the topology of convergence on members of $\Phi, F$ having the topology of convergence on members of $\Psi$, will consist of sets of the following construction:

$$
\begin{aligned}
W(U, \mathscr{F})= & \{T: T \text { in } G, \text { and there is an } A \text { in } \mathscr{F} \text { such that } T(A) \subset U\} \\
= & \{T: T \text { in } G \text { and there is an } A \text { in } \mathscr{F} \text { such that for each } x \text { in } A \text { there exists } \\
& \text { a } \left.B_{x} \text { in } \mathscr{G} \text { with the property that }\left|\left\langle T(x), y^{\prime}\right\rangle\right| \leqq \varepsilon \text { for all } y^{\prime} \text { in } B_{x}\right\} .
\end{aligned}
$$

Next we look at $G$ as a space of linear forms, and examine the sets which form a subbase for the neighborhoods of 0 for the topology of convergence on members of $\Phi \mid \Psi$. Such sets are of the form $W_{0}(\varepsilon, \mathscr{F} \mid \mathscr{G})=\{T: T$ in $G$ and there is an $A$ in $\mathscr{F}$ with a map $\alpha$ of $A$ into $\mathscr{G}$ such that $\left|T\left(x \otimes y^{\prime}\right)\right|=\left|\left\langle T(x), y^{\prime}\right\rangle\right| \leqq \varepsilon$ for all $y^{\prime}$ in $\alpha(x)$ and all $x$ in $A$. By setting $\alpha(x)=B_{x}$ which was obtained in the definition of $W(U, \mathscr{F})$, we see that $W_{0}(\varepsilon, \mathscr{F} \backslash \mathscr{G})$ is $W(U, \mathscr{F})$. 
(vi) The proof of this part is analogous to (v).

(vii) The $\Psi$-topology on $F$ is the topology of uniform convergence on members of $B$ and the $\Phi$-topology on $G$ is uniform convergence on members of $A$ [3], [5]. From (v) it is seen that this is the $\Phi \mid \Psi$-topology on the linear forms. Two applications of (iii) make it possible to conclude that $\Phi \otimes \Psi=\Phi|\Psi=\Psi| \Phi$. Thus the topologies are the same.

The situation in part (iii) of the above proposition occurs when $F$ is topologized by uniform convergence on a family of subsets of $F^{\#}$. The situation in (iv) is present when the space $G$ of linear operators is topologized by pointwise convergence on $E$, independent of how $F$ is topologized. The dual situation occurs when $F$ has the $\sigma\left(F, F^{\prime}\right)$-topology.

In Remark 4.5 there are examples where the $\Phi \mid \Psi$-topology and the $\Psi \mid \Phi$ topology are not the same. In fact, they produce different dual spaces.

Once a topology on $G$ has been represented as convergence on a family of filters from $E \otimes F^{\#}$, the weak topology, i.e. the $\sigma\left(G, G^{\prime}\right)$-topology, is immediately obtained by means of Theorem 2 of [5]. When Theorem 1.4 is used, the resulting topology is a weak topology. This makes it necessary to raise the converse question and ask what stronger topologies give the same dual space. The following theorem and corollary are concerned with such situations.

3.3. Theorem. Let $G$ be a linear space of linear maps of $E$ into $F$. The space $G$ will also be viewed as a collection of linear forms defined on $E \otimes F^{\#}$. All topologies are assumed to be linear.

(i) If the space $G$ of linear operators is given the $\Phi$-topology, $F$ having the $\Psi$ topology, then the $\sigma\left(G, G^{\prime}\right)$-topology is convergence on all ultrafilters which are refinements of the convex hulls of members of $\Phi \mid \Psi$ (see Theorem 2 of [5]).

(ii) If the space $G$ of linear operators is given the $\Phi$-topology, $F$ having the $\sigma\left(F, F^{\#)-}\right.$ topology, then the $\sigma\left(G, G^{\prime}\right)$-topology is the operator space topology of convergence on all ultrafilters which are refinements of the convex hulls of members of $\Phi$. (Note the similarity of this statement for vector valued functions to Theorem 2 of [5] for scalar valued functions.)

(iii) If $F$ has a locally convex topology with dual space $F^{\prime}$, and the operator space $G$ is given the topology of pointwise convergence on $E$, then the $\sigma\left(G, G^{\prime}\right)$-topology is pointwise convergence on $E, F$ having the $\sigma\left(F, F^{\prime}\right)$-topology. (These are the strong and weak operator topologies. See pp. 475-476 of [6].)

(iv) Let $E, F, E^{\prime}, F^{\#}, A$ and $B$ be defined as in Lemma 2.1. If the subspace $G$ of $L(E, F)$ is given the topology of almost uniform convergence on members of $\mathscr{A}, F$ having the topology of uniform convergence on members of $\mathscr{B}$, and $H$ is the subspace of $G$ consisting of all linear operators whose restrictions to members of $\mathscr{A}$ are $\sigma\left(E, E^{\prime}\right)$-continuous, then on the linear forms the topology of almost uniform convergence on members of $\mathscr{A} \otimes \mathscr{B}$ is a weak topology which coincides with the $\sigma\left(G, G^{\prime}\right)$ topology on $H$ and is weaker than the $\sigma\left(G, G^{\prime}\right)$-topology on $G$. 
(v) If the space $G$ of linear operators has the topology of uniform convergence on members of $\mathscr{A}, F$ having the topology of uniform convergence on members of $\mathscr{B}$ (see Lemma 2.1 for notation), then the $\sigma\left(G, G^{\prime}\right)$-topology is almost uniform convergence on the convex hulls of members of $\mathscr{A} \otimes \mathscr{B}$. (A special case of this is the norm topology on the bounded linear operators which map a normed space into a normed space.)

Proof. (i) The result follows from (v) of 3.2 and Theorem 2 of [5].

(ii) Let $\Psi$ be the family of filters in $F^{\#}$ having as a basis a subset of $F^{\#}$ consisting of a single point. The $\sigma\left(F, F^{\#)}\right.$ )-topology is convergence on members of $\Psi$ (see Proposition 2 of [5]). On the linear operators $G$ the topology of convergence on members of $\Phi$ is the same as the topology on the linear forms that is obtained by convergence on members of $\Phi \otimes \Psi$ (see 4.2(iii)). Each member $\mathscr{F} \otimes \mathscr{G}$ of $\Phi \otimes \Psi$ has a basis of the form $\left\{A \otimes y^{\prime}: A\right.$ in $\left.\mathscr{F}\right\}$ where $y^{\prime}$ is a basis for $\mathscr{G}$. Thus, every ultrafilter which is a refinement of a convex hull of a member of $\Phi \otimes \Psi$ must have a basis of the form $\left\{C \otimes\left(y^{\prime}\right)\right.$ : the set $C$ ranging through the basis for an ultrafilter which is a refinement of the convex hull of a member of $\Phi\}$. The topology of convergence on all ultrafilters which are refinements of convex hulls of members of $\Phi \otimes \Psi$ becomes convergence on a family of filters obtained by taking the tensor product of all ultrafilters which are refinements of convex hulls of members of $\Phi$ with the original members of $\Psi$. We use parts (iii) and (v) of Proposition 3.1 to conclude that the $\sigma\left(G, G^{\prime}\right)$-topology is the operator space topology of convergence on all ultrafilters which are refinements of convex hulls of members of $\Phi, F$ having the $\sigma\left(F, F^{\prime}\right)$-topology.

(iii) Let $\Psi$ be the family of filters in $F^{\prime}$, each filter having a single equicontinuous subset of $F^{\prime}$ as a basis. The topology on $F$ is convergence on members of $\Psi$ [5, Proposition 2]. Let $\Phi$ be the family of filters in $E$ which have as a basis the sets consisting of a single point of $E$. The topology of pointwise convergence on $E$ is convergence on members of $\Phi$. We use the same line of argument as in the proof of part (ii) to see that the $\sigma\left(G, G^{\prime}\right)$-topology is pointwise convergence on $E, F$ having the topology of convergence on all ultrafilters which are refinements of convex hulls of members of $\Psi$, which is nothing more than the $\sigma\left(F, F^{\prime}\right)$-topology on $F$ [5, Theorem 2].

(iv) Let $\Phi$ be all ultrafilters containing a member of $\mathscr{A}$. Convergence on members of $\Phi$ is the same as almost uniform convergence on members of $\mathscr{A}$ (see Corollary 2.4 of [3]). Let $\Psi$ be the family of filters of subsets of $F$, each filter having a single member of $\mathscr{B}$ as a basis. We use (v) and (iii) of Proposition 3.2 to see that the topology on $G$ is convergence on members of $\Phi \otimes \Psi$.

Almost uniform convergence on members of $A \otimes B$ is a weak topology on $G$ (Proposition 4 of [5]). It is the same as convergence on all ultrafilters which contain a member of $\mathscr{A} \otimes \mathscr{B}$. In contrast the $\sigma\left(G, G^{\prime}\right)$-topology is convergence on all ultrafilters which are refinements of the convex hull of some member of $\Phi \otimes \Psi$. Since the $\sigma\left(G, G^{\prime}\right)$-topology is obtained as convergence on a larger family of ultra- 
filters, the topology of almost uniform convergence on members of $\mathscr{A} \otimes \mathscr{B}$ must be a weaker topology.

The linear forms which make up $H$ have restrictions to members of $\mathscr{A} \otimes \mathscr{B}$ which are $\sigma\left(E \otimes F^{\#}, E^{\prime} \otimes F\right)$-continuous (Lemma 2.1). We use part (iv) of Lemma 2.1 to extend the linear forms over the compact members of $\{\bar{A} \otimes \bar{B}: A \in \mathscr{A}, B \in \mathscr{B}\}$. On $H$ the topology of almost uniform convergence on members of $\mathscr{A} \otimes \mathscr{B}$ is pointwise convergence on members of $\{\bar{A} \otimes \bar{B}: A \in \mathscr{A}$, $B \in \mathscr{B}\}$ due to the continuity of members of $H$ [1], [3]. The adherence of each member of $\Phi \otimes \Psi$ is a convex set of the form $\{x\} \otimes \bar{B}$. Thus, the ultrafilters which are refinements of the convex hulls will converge to points in $\{x\} \otimes \bar{B}$. Due to the continuity of the members of $H$ we see that convergence on ultrafilters which are refinements of convex hulls of members of $\Phi \otimes \Psi$ is the same as pointwise convergence on members of $\{\bar{A} \otimes \bar{B}: A \in \mathscr{A}, B \in \mathscr{B}\}$. This is the topology of almost uniform convergence on members of $\mathscr{A} \otimes \mathscr{B}$. We see that this is the $\sigma\left(G, G^{\prime}\right)$ topology when restricted to $H$.

(v) The topology on $G$ is convergence on all filters having as a basis a member of $\mathscr{A} \otimes \mathscr{B}$. All convex hulls of such filters are all filters having the convex hull of a member of $A \otimes B$ as a basis. Convergence on all ultrafilters which are refinements becomes almost uniform convergence on the convex hulls of members of $\mathscr{A} \otimes \mathscr{B}$.

3.4. Corollary. Let $E$ and $F$ be Banach spaces, $S$ the unit ball of $E, S^{\prime}$ the unit ball of the dual space $F^{\prime}$, and $H$ a linear space of bounded linear operators with the norm topology. A filter $\mathscr{P}$ in $H$ converges to 0 for the $\sigma\left(H, H^{\prime}\right)$-topology if and only if

(i) $\mathscr{P}$ converges to 0 for the weak operator topology, and

(ii) for every set $P$ in a refinement of $\mathscr{P}$ and every positive number $\varepsilon$ there exists a finite subset $\left(T_{1}, \ldots, T_{k}\right)$ of $P$ such that

$$
\min _{i}\left|\sum_{j=1}^{n} a_{j}\left\langle T_{i}\left(x_{j}\right), x_{j}^{\prime}\right\rangle\right| \leqq \varepsilon
$$

for every triple of finite sets $\left(x_{1}, \ldots, x_{n}\right) \subset S,\left(y_{1}^{\prime}, \ldots, y_{n}^{\prime}\right) \subset S^{\prime}$ and positive scalars $\left(a_{1}, \ldots, a_{n}\right)$ such that $\sum_{j=1}^{n} a_{j}=1$.

Proof. The corollary is a special case of part (v) of the theorem. It is obtained by using the fact that almost uniform convergence on the convex hull of $S \otimes S^{\prime}$ is equivalent to pointwise convergence and quasi-uniform convergence of all refinements of the convex hull of the filter $P$ (see Definition 2.3 of [1]).

Part (iv) of Theorem 3.3 has a dual version in which $G$ is replaced by the transpose of its members (see p. 254 of [8]). The members of $\mathscr{A}$ are assumed to be convex and the rolls of $\mathscr{A}$ and $\mathscr{B}$ are interchanged with the appropriate change in $H$. When we view the operators as linear forms we see that there nas been little change. The desired weak topology is still almost uniform convergence on members of $\mathscr{A} \otimes \mathscr{B}$. 
When Theorem 1.4 is applied to the linear form characterizations of the linear operators (see Theorem 2.4) we obtain the topology of almost uniform convergence on the members of a family $D$. The family $D$ has been constructed from several other families. The following lemmas will be useful in simplyfying the situation.

3.5. Lemma. Let $\mathscr{D}_{1}$ and $\mathscr{D}_{2}$ be two families of balanced subsets of $E \otimes F^{\#}$, both families being closed under finite sums. On a subspace $G$ of $\left(E \otimes F^{\#}\right)^{*}$ the locally convex linear topology of almost uniform convergence on members of $\mathscr{D}_{1}+\mathscr{D}_{2}$ is the supremum of the topologies of almost uniform convergence on $\mathscr{D}_{1}$ and on $\mathscr{D}_{2}$.

Proof. We use Proposition 2 of [5] to see that the topology of almost uniform convergence on the members of a family of $\mathscr{D}_{1}+\mathscr{D}_{2}$ is pointwise convergence on the members of a family of subsets of $G^{*}$. The family is composed of the $\sigma\left(G^{*}, G\right)$-closure of the images of members of $\mathscr{D}_{1}+\mathscr{D}_{2}$. We consider only the natural map of $E \otimes F^{\#}$ into $G^{*}$. The same thing can be done for the families $\mathscr{D}_{1}$ and $\mathscr{D}_{2}$. This leaves us with three families, $\overline{\mathscr{D}}_{1}, \overline{\mathscr{D}}_{2}$, and $\mathrm{Cl}\left(\mathscr{D}_{1}+\mathscr{D}_{2}\right)$ of subsets of $G^{*}$. All the subsets are compact because the topologies are linear (see the proof of Proposition 2 of [5]). From this we see that $\overline{\mathscr{D}}_{1}+\overline{\mathscr{D}}_{2}=\mathrm{Cl}\left(\mathscr{D}_{1}+\mathscr{D}_{2}\right)$. Thus, the topology of pointwise convergence on members of $\mathrm{Cl}\left(\mathscr{D}_{1}+\mathscr{D}_{2}\right)$ is the supremum of the topologies of pointwise convergence on members of $\overline{\mathscr{D}}_{1}$ and on members of $\overline{\mathscr{D}}_{2}$. The same statement can be made for the topologies of almost uniform convergence.

3.6. LEMMA. If $X$ is a linear space with two distinct weak topologies $\sigma_{1}$ and $\sigma_{2}$ then $\sigma_{1} \vee \sigma_{2}$ is a weak topology and $\mu_{1} \vee \mu_{2}$ gives the same dual space as $\sigma_{1} \vee \sigma_{2}$ for every $\mu_{i}$ which gives the same dual space as $\sigma_{i}$ for $i=1,2$.

Proof. Let $X_{1}^{\prime}$ be the dual of $X$ for the $\sigma_{1}$-topology and let $X_{2}^{\prime}$ be the dual of $X$ for the $\sigma_{2}$-topology. Consider $X_{1}^{\prime}$ and $X_{2}^{\prime}$ as subsets of $X^{*}$ and let $X^{\prime}$ be the linear span of $X_{1}^{\prime} \cup X_{2}^{\prime}$. Since the $\sigma_{1} \vee \sigma_{2}$-topology is pointwise convergence on $X^{\prime}$, the $\sigma_{1} \vee \sigma_{2}$-topology is a weak topology with dual space $X^{\prime}$. A topology $\mu_{i}$ having the same dual as the $\sigma_{i}$-topology can be represented as uniform convergence on a family $\mathscr{A}_{i}$ of $\sigma\left(X^{\prime}, X\right)$-compact, convex, balanced subsets of $X_{i}^{\prime} \subset X^{\prime}$, for $i=1,2$. Thus the $\mu_{1} \vee \mu_{2}$-topology is uniform convergence on the members of $\mathscr{A}_{1}+\mathscr{A}_{2}$ which is a family of $\sigma\left(X^{\prime}, X\right)$-compact, convex, balanced subsets covering $X^{\prime}$. Since the Mackey topology related to the $\sigma_{1} \vee \sigma_{2}$-topology is a stronger topology of uniform convergence on all $\sigma\left(X^{\prime}, X\right)$-compact, convex subsets of $X^{\prime}$, we see that the $\mu_{1} \vee \mu_{2}$-topology gives the same dual space as the $\sigma_{1} \vee \sigma_{2}$-topology.

4. The approximation topologies. We now return to the original problem. Applications of Theorems 1.1 and 1.4 produce approximation topologies for the classes of linear forms defined in Theorem 2.4. Theorem 3.3 and Lemmas 3.5 and 3.6 produce additional topologies. When possible we formulate the topologies on a space of linear operators. This outlines the method of this paper which is to carry a problem from the space of linear operators into a space of linear forms, 
attack the problem in the space of linear forms and then return to the linear operators.

The following theorem is a continuation of Theorem 2.4:

4.1. TheOREM. Let $E$ and $F$ be normed linear spaces and set $N=E^{\prime} \otimes F$. The linear spaces $H$ and $G$ are specified in each part. The respective topologies are placed on $G$ in order that $\bar{N}=H$ or $\hat{N}=H$.

(i) Linear operators.

$H=$ all linear operators of $E$ into $F$.

$G=$ all linear operators of $E$ into $F^{\prime \prime}$.

$\hat{N}=H$ for the strong operator topology on $G$.

$\bar{N}=H$ for the weak operator topology which is the $\sigma\left(G, G^{\prime}\right)$ topology on $G$.

(ii) Continuous linear operators.

$H=G=$ all continuous linear operators of $E$ into $F$.

$\hat{N}=H$ for the topology which is the supremum of the strong operator topology and the topology of uniform convergence on the unit ball of $E, \hat{F}$ having the $\sigma\left(\hat{F}, F^{\prime}\right)$ topology.

$\hat{N}=H$ for the topology which is the supremum of the topology of pointwise convergence on $\hat{E}$ (for the continuous extensions), $\hat{F}$ having the norm topology, and the topology of uniform convergence on the unit ball of $E, \hat{F}$ having the $\sigma\left(\hat{F}, F^{\prime}\right)$-topology.

(iii) Weakly compact operators.

$H=$ all weakly compact operators mapping $E$ into $\hat{F}$.

$G=$ all continuous linear operators mapping $E$ into $F$.

$\hat{N}=H$ for the topology which is the supremum of the topology of uniform convergence on the unit ball of $E, \hat{F}$ having the $\sigma\left(F, F^{\prime}\right)$-topology and the $\Psi \mid \Phi$ topology where $\Psi$ is all filters containing the unit ball of $F^{\prime}$ and $\Phi$ is all ultrafilters containing the unit ball of $E$ (see 3.2(vi)).

$\bar{N}=H$ for the $\sigma\left(G, G^{\prime}\right)$-topology which is again a $\Psi \mid \Phi$ topology where $\Psi$ is all ultrafilters containing the unit ball of $F^{\prime}$ and $\Phi$ is all ultrafilters containing the unit ball of $E$.

When all members of $G$ are replaced by their second adjoints, the $\sigma\left(G, G^{\prime}\right)$-topology is pointwise convergence on $E^{\prime \prime}, F^{\prime \prime}$ having the $\sigma\left(F^{\prime \prime}, F^{\prime \prime}\right)$-topology.

(iv) Completely continuous linear operators.

$H=$ all continuous linear operators mapping weakly compact sets of $E$ into compact subsets of $\hat{F}$.

$G=$ all continuous linear operators of $E$ into $\hat{F}$.

$\bar{N}=H$ for

(a) the topology of almost uniform convergence on members of $K^{2}, \hat{F}$ having the norm topology;

(b) the $\Psi \backslash \Phi$ topology where $\Psi$ is all ultrafilters containing the unit ball of $F^{\prime}$ and $\Phi$ is all filters containing a member of $K^{2}$;

(c) the supremum of (a) and (b); 
(d) the topology of almost uniform convergence on members of $K^{2} \otimes S^{\prime}$ (this is the $\sigma\left(H, H^{\prime}\right)$-topology when the topologies of (a), (b) and (c) are placed on $\left.H\right)$;

(e) the supremum of any of the topologies from (a), (b), (c), and (d) with the topologies of part (ii).

(v) Compact linear operators.

$H=$ all compact linear operators mapping $E$ into $\hat{F}$.

$G=$ all continuous linear operators mapping $E$ into $\hat{F}$.

$\bar{N}=H$ for

(a) the topology of almost uniform convergence on the unit ball of $E, \hat{F}$ having the norm topology (see Theorem 1 of [2]);

(b) the $\Psi \backslash \Phi$ topology where $\Psi$ is all ultrafilters containing the unit ball of $F^{\prime}$ and $\Phi$ is all filters containing the unit ball of $E$ (see 3.2(vi));

(c) the supremum of (a) and (b);

(d) almost uniform convergence on members of $S \otimes S^{\prime}$ (this is the $\sigma\left(H, H^{\prime}\right)$ topology when the topologies of (a), (b), and (c) are restricted to $H)$;

(e) the supremum of the topologies of (a), (b), (c), and (d) with any of the topologies from (ii), (iii), and (iv).

Proof. Although the results of part (i) are known, our proof serves as an introduction to the methods that are used.

(i) The members of $H$ are viewed as all linear forms whose restrictions to members of $J \otimes S^{\prime}$ are $\sigma\left(E \otimes F^{\prime}, E^{\prime} \otimes F\right)$-continuous (see Theorem 2.4(i)). The family $J \otimes S^{\prime}$ contains the convex hulls of all its members. This makes it possible to use Theorem 1.1. Thus the completion of $N$ is $H$ for the topology of uniform convergence on members of $J \otimes S^{\prime}$. When we return to the linear operators we see that this is the strong operator topology (see 3.2(vii)). The $\sigma\left(G, G^{\prime}\right)$-closure of $N$ is also $H$ because $N$ is a convex set. In this case, the $\sigma\left(G, G^{\prime}\right)$-topology is the weak operator topology (see Theorem 3.3(iii)).

(ii) We follow the same line of reasoning as above to see that the continuous linear operators are the completion of $E^{\prime} \otimes F$ for the topology of uniform convergence on the members of $\left(J \otimes S^{\prime}\right)+\left(S \otimes J^{\prime}\right)$. When we view $G$ as a space of linear operators, the topology becomes the supremum of the strong operator topology and the topology of uniform convergence on the unit ball of $E, \hat{F}$ having the $\sigma\left(\hat{F}, F^{\prime}\right)$-topology. From Lemma 3.6 we see that the $\sigma\left(G, G^{\prime}\right)$-topology is the supremum of two topologies. One is the weak operator topology (3.3(iii)) and the other is almost uniform convergence on the unit ball of $E, \hat{F}$ having the $\sigma\left(\hat{F}, F^{\prime}\right)$ topology (3.3(ii)). The latter is the supremum because it is a stronger topology. When the members of $G$ are replaced by their second adjoints, the resulting linear operators are $\sigma\left(E^{\prime \prime}, E^{\prime}\right)$-continuous, $F^{\prime \prime}$ having the $\sigma\left(F^{\prime \prime}, F^{\prime}\right)$-topology. We recall that the unit ball of $E^{\prime \prime}$ is $\sigma\left(E^{\prime \prime}, E^{\prime}\right)$-compact and the unit ball of $E$ is a dense subset. Thus the topology of almost uniform convergence on the unit ball of $E$ becomes pointwise convergence on the unit ball of $E^{\prime \prime}$ (see Theorems 4.1 and 4.2 of [1]). The 
remainder of part (ii) is obtained by continuously extending the operators over $\hat{E}$ and repeating the above arguments.

(iii) We use the same argument again to see that $\hat{N}=H$ for a topology which is the supremum of the topology of uniform convergence on members of $J^{\prime \prime} \otimes S^{\prime}$ and the topology of uniform convergence on members of $S \otimes J^{\prime}$. We now consider $G$ as naturally embedded in $L\left(E^{\prime \prime}, F^{\prime \prime}\right)$. Uniform convergence on $J^{\prime \prime} \otimes S^{\prime}$ is the strong operator topology and the other topology is uniform convergence on the members of $S$ embedded in $E^{\prime \prime}, F^{\prime \prime}$ having the $\sigma\left(F^{\prime \prime}, F^{\prime}\right)$-topology. Next, we consider the subspace of $L\left(F^{\prime}, E^{\prime}\right)$ which is composed of all the adjoints of members of $G$. The first of the above topologies becomes uniform convergence on the unit ball of $F^{\prime}, E^{\prime}$ having the $\sigma\left(E^{\prime}, E^{\prime \prime}\right)$-topology. This is a $\Psi \backslash \Phi$ topology on $G$ where $\Phi$ is all ultrafilters containing a member of $S$ and $\Psi$ is all filters containing the unit ball of $F^{\prime}$ (see 3.2(vi)). Note that the $\sigma\left(E^{\prime}, E^{\prime \prime}\right)$-topology is convergence on members of $\Phi$ (Corollary 5.7 [1]). The other topology is uniform convergence on the unit ball of $E, \hat{F}$ having the $\sigma\left(\hat{F}, F^{\prime}\right)$-topology.

Lemma 3.6 tells us that the $\sigma\left(G, G^{\prime}\right)$-topology is the supremum of two weak topologies. But it is only necessary to consider the weak topology related to the above $\Psi \mid \Phi$-topology because it is stronger than the other weak topology. We view the operators as members of $L\left(F^{\prime}, E^{\prime}\right)$ and use Theorem 3.3(ii). Thus the $\sigma\left(G, G^{\prime}\right)$ topology is a new $\Psi \mid \Phi$-topology where $\Psi$ becomes all ultralfiters containing the unit ball of $F^{\prime}$, and $\Phi$ is unchanged. When we embed $G$ in $L\left(E^{\prime \prime}, F^{\prime \prime}\right)$ this becomes the topology of pointwise convergence on $E^{\prime \prime}, F^{\prime \prime}$ having the $\sigma\left(F^{\prime \prime}, F^{\prime \prime}\right)$-topology. This is seen by means of Corollary 5.7 of [1] and our Proposition 3.2(iii).

(iv) We use Theorem 1.4 to see that $\bar{N}=H$ for the weak topology of almost uniform convergence on members of $K^{2} \otimes S^{\prime}$. Thus we have the topology of part (d). Part (a) is obtained by applying 3.3(iv). On $G$ the weak topology related to the topology of part (a) is stronger than the topology of part (d), but they are identical when restricted to $H$. Thus $\bar{N}=H$ for these topologies on $G$. We view the operators as members of $L\left(F^{\prime}, E^{\prime}\right)$ and apply the same argument to obtain part (b).

The weak topologies related to the topologies of parts (a) and (b) are identical when restricted to $H$. Thus the same will be true for the topology which is the supremum of those in (a) and (b) (Lemma 3.6). This establishes part (c).

We have also characterized the space $H$ as all linear forms whose restrictions to members of $\left(K^{2} \otimes S\right)+\left(S \otimes J^{\prime}\right)$ are continuous $(2.4(\mathrm{iv}(\mathrm{b})))$. By Theorem 1.4, $\bar{N}=H$ for the topology of almost uniform convergence on the members of $\left(K^{2} \otimes S^{\prime}\right)+\left(S \otimes J^{\prime}\right)$. This is the supremum of the topologies of almost uniform convergence on $K^{2} \otimes S^{\prime}$ and on $S \otimes J^{\prime}$ (Lemma 3.5). The first topology is the topology of part (d) and the latter topology is the weak topology of part (ii). Lemma 3.6 leads to the results stated in (e).

(v) Parts (a), (b), (c), and (d) are obtained by the same line of reasoning used in part (iv). Because the weak topology of part (d) is stronger than the weak topologies in (ii) through (iv), the topologies of (e) continue to make $\bar{N}=H$ (Lemma 3.6). 
4.2. Remark. In part (ii) and (iii) of the above theorem the space $G$ may be replaced by a larger class of operators. In (ii) we could have used all linear maps of $E$ into $F^{\prime \prime}$ and in (iii) it could have been all continuous linear maps of $E^{\prime \prime}$ into $F^{\prime \prime}$. There would be some change in the formulation of the weak topologies.

4.3. REMARK. The subclass of the strictly singular operators presented in 2.5 can be obtained as the closure of $E^{\prime} \otimes F$ for the supremum of any two topologies from (iii) and (iv) respectively.

4.4. Remark. In the space of all continuous linear operators mapping $E$ into $F$ the collection of bounded sets is the same for the norm topology and the topologies of parts (ii), (iii) and (v). This is because the topologies are weaker than the norm topology and stronger than the topology of almost uniform convergence on the unit ball of $E, F$ having the $\sigma\left(F, F^{\prime}\right)$-topology. The bounded sets for the latter topology are the same as for the norm topology. This can be seen by using the uniform boundedness theorem on the second adjoints of the operators, or by means of Theorem 1 of [4].

4.5. REMARK. The topology of $(\mathrm{v}(\mathrm{a}))$ is a $\Phi \mid \Psi$-topology where $\Phi$ is all ultrafilters containing the unit ball of $E$ and $\Psi$ is all filters containing the unit ball of $F^{\prime}$. The $\Psi \mid \Phi$ topology of the same family of filters is the first topology of part (iii). The $\Phi \mid \Psi^{\circ}$-topology is the topology $\alpha$ of Theorem 1 in [2]. Theorem 2 of the same paper is not correct but can be corrected by using the $\Psi \backslash \Phi$-topology in place of the topology $\beta$.

Now consider $\Psi$ to be all ultrafilters containing the unit ball of $F^{\prime}$ and $\Phi$ as above. The $\Phi \backslash \Psi$-topology is the weak topology of part (ii) and is also the erroneous topology $\beta$ of reference [2]. In comparision, the topology is the weak topology of part (iii). Thus this $\Psi \backslash \Phi$-topology could also be used to correct Theorem 2 of [2].

4.6. Remark. When $E$ and $F$ are locally convex spaces, Theorem 4.1 can be generalized by replacing $S$ by the family of bounded subsets of $E$ and $S^{\prime}$ by the equicontinuous subsets of $F^{\prime}$. The results are not as pretty because many of the operators needed to form the completions are not continuous.

4.7. Theorem. Let $E$ and $F$ be Banach spaces.

(a) A compact operator $T$ can be approximated in norm by continuous linear operators of finite-dimensional range if and only if the linear form which $T$ defines on $E \otimes F^{\prime}$ is $\sigma\left(E \otimes F^{\prime}, E^{\prime} \otimes F\right)$-continuous when restricted to the convex hull of $S \otimes S^{\prime}, S$ and $S^{\prime}$ being the unit balls of $E$ and $F^{\prime}$ respectively.

(b) The compact operators are the completion of $E^{\prime} \otimes F$ for the norm topology if and only if every linear form whose restriction to $S \otimes S^{\prime}$ is $\sigma\left(E \otimes F^{\prime}, E^{\prime} \otimes F\right)$ continuous is continuous when restricted to the convex hull of $S \otimes S^{\prime}$.

The proof follows from parts (v) and (vi) of Theorem 2.4 and Theorem 1.1.

4.8. Theorem. (The Approximation Property, see Proposition 35 on p. 164 of [7].) Let $E$ be a locally convex space. The following statements are equivalent:

(a) The space $E$ has the approximation property. 
(b) The space $E^{\prime} \otimes E$ is dense in the space of all continuous linear operators mapping $E$ into $E$ for the topology of uniform convergence on precompact subsets of $E$.

(c) The natural linear form $\left(x \otimes x^{\prime} \mapsto\left\langle x, x^{\prime}\right\rangle\right)$ on $E \otimes E^{\prime}$ has restrictions to the convex hulls of members of $\mathscr{A} \otimes \mathscr{B}$ which are $\sigma\left(E \otimes E^{\prime}, E^{\prime} \otimes E\right)$-continuous. The family $\mathscr{A}$ is all precompact subsets of $E$ and $\mathscr{B}$ is all equicontinuous subsets of $E^{\prime}$.

(d) For every locally convex space $F$ and continuous linear operator $T$ mapping $E$ into $F$, the linear form which $T$ defines on $E \otimes F^{\prime}$ has restrictions to the convex hulls of members of $\mathscr{A} \otimes \mathscr{B}$ which are $\sigma\left(E \otimes F^{\prime}, E^{\prime} \otimes F\right)$-continuous. The family $\mathscr{A}$ is all precompact subsets of $E$ and $\mathscr{B}$ is all equicontinuous subsets of $F^{\prime}$.

(e) For every locally convex space $F$ and every weakly continuous linear operator $T$ of $F$ into $E$ which maps a family $\mathscr{A}$ of bounded subsets of $F$ into precompact subsets of $E$, the linear form $T$ is $\sigma\left(F \otimes E^{\prime}, F^{\prime} \otimes E\right)$-continuous when restricted to the convex hulls of members of $\mathscr{A} \otimes \mathscr{B}$. The family $\mathscr{B}$ is all equicontinuous subsets of $E^{\prime}$.

When $E$ is a Banach space, the above statements are equivalent to the following:

(f) For every Banach space $F, S$ its unit ball and $S^{\prime}$ the unit ball of $E^{\prime}$, every linear form whose restriction to $S \otimes S^{\prime}$ is $\sigma\left(F \otimes E^{\prime}, F^{\prime} \otimes E\right)$-continuous is also continuous when restricted to the convex hull of $S \otimes S^{\prime}$.

Proof. The equivalence of (a) and (b) can be found on p. 165 of [7]. With the help of Theorem 1.1 we see that statement (c) is equivalent to saying that the identity of $E$ onto $E$ is in the completion of $E^{\prime} \otimes E$ for the topology of part (b). This is shown to be equivalent to (b) in the reference cited. In the same way (d) is equivalent to saying that every continuous operator is in the completion of $E^{\prime} \otimes F$ for the topology of part (b). The equivalence to this new form of (d) to (b) can be found in the reference cited.

Statement (e) implies (c). For the converse let $T$ be a weakly continuous linear operator of $F$ into $E$ such that $T(A)$ is precompact for every $A$ in the family $\mathscr{A}$. For a fixed $A$ from $\mathscr{A}$ and a neighborhood $V$ of 0 in $E$, our equivalent statement of (c) obtained from Theorem 1.1 gives the existence of a mapping $P$ in $E^{\prime} \otimes E$ such that $T(x)-P \circ T(x) \in V$ for all $x$ in $A$. The mapping $P \circ T$ is in $F^{\prime} \otimes E$. Thus $T$ is in the completion of $F^{\prime} \otimes E$ for the topology of uniform convergence on members of $\mathscr{A}$. When viewed as linear forms this is the topology of uniform convergence on members of $\mathscr{A} \otimes \mathscr{B}$. Theorem 1.1 is used to conclude that the restrictions of $T$ to the convex hulls of members of $\mathscr{A} \otimes \mathscr{B}$ are $\sigma\left(F \otimes E^{\prime}, F^{\prime} \otimes E\right)$-continuous.

In Theorem 4.7 we showed that statement (f) is equivalent to the compact operators being the completion of $F^{\prime} \otimes E$ for the norm topology. On p. 165 of [7] it is stated that this is equivalent to statement (b) when $E$ is a Banach space.

4.9. Remark. Statement (f) of the above theorem suggests that we should consider the possibility of constructing a Banach space $E$ which has a linear form defined on $E \otimes E^{\prime}$ such that the restriction of the linear form to $S \otimes S^{\prime}$ is $\sigma\left(E \otimes E^{\prime}, E \otimes E\right)$-continuous, but whose restriction to the convex hull of $S \otimes S^{\prime}$ is not continuous. If there is such a Banach space it will be a counterexample of 
both the basis problem and the compact operator problem (see pp. 6 and 12 of [11]).

4.10. Remark. In part (b) of Theorem 4.8 the space $G$ of all continuous operators is given the topology of uniform convergence on precompact subsets of $E$. In the context of Theorems 4.1 and 2.4 this is the topology of uniform convergence on members of $K^{0}$. Part (b) of Theorem 4.8 (when it is true) implies that $G=H \subset \bar{N}$ for this topology. Part (ii) of Theorem 4.1 also gives a topology for which this is true, but the two topologies may not produce the same dual space. This can be seen by comparing the related weak topologies (use part (v) of 3.3). An exception is given in the following corollary.

4.11. Corollary to Theorem 4.8. Every locally convex space $E$ with the $\sigma\left(E, E^{\prime}\right)$-topology has the approximation property. (In reference [12] Ivan Singer noted that this is true for reflexive spaces.)

Proof. Let $A$ denote the collection of all bounded sets (=all precompact sets) in $E$ and let $J^{\prime}$ be the convex hulls of all finite-dimensional subsets of $E^{\prime}$. From a linear point of view, the topology of part (b) of 4.8 is uniform convergence on members of $A \otimes J^{\prime}$. Use Remark 4.6 to formulate 2.4 and 4.1 in the present situation. In place of part (ii) of 4.1 we have that $G=H \subset \bar{N}$ for the topology of uniform convergence on member $\left(A \otimes J^{\prime}\right)+\left(J+J^{\prime}\right)$. But $\left(A \otimes J^{\prime}\right)+\left(J \otimes J^{\prime}\right)$ is $A \otimes J^{\prime}$, which verifies part (b) of 4.8 .

When a space has the approximation property, it is possible to improve on the linear form characterizations of the linear operators. This is done by applying parts (d) and (e) of Theorem 4.8 to the characterizations of Theorem 2.4. This has the effect of enlarging the families $K^{0} \otimes S^{\prime}, K^{1} \otimes S^{\prime}, K^{2} \otimes S^{\prime}$, and $S \otimes S^{\prime}$ by including in them the convex hulls of their members. Topologies are now obtained by means of Theorem 1.1. The following theorem is a continuation of 4.1 .

4.12. TheOREM. Let $E$ and $F$ be normed spaces and $G$ all continuous linear maps of $E$ into $\hat{F}$.

(i) Let $H$ be all continuous linear maps of $E$ into $\hat{F}$. If either $E$ or $F$ has the approximation property, then $\hat{N}=H$ for the topology which is the supremum of the topology of uniform convergence on the precompact subsets of $E, \hat{F}$ having the norm topology, and the topology of uniform convergence on the unit ball of $E, \hat{F}$ having the $\sigma\left(\hat{F}, F^{\prime}\right)$ topology.

(ii) Let $H$ be all weakly compact operators mapping $E$ into $\hat{F}$. If $F$ has the approximation property then $\hat{N}=H$ for the topology which is the supremum of the topology of uniform convergence on members of $K^{\prime}, \hat{F}$ having the norm topology, and the $\Psi \backslash \Phi$ topology where $\Psi$ is all filters containing the unit ball of $F^{\prime}$ and $\Phi$ is all ultrafilters containing the unit ball of $E$. (This may be made even stronger by taking the supremum with the topology of uniform convergence on the unit ball of $E, \hat{F}$ having the $\sigma\left(\hat{F}, F^{\prime}\right)$ topology.)

(iii) Let $H$ be all completely continuous linear operators mapping $E$ into $\hat{F}$. If $F$ has the approximation property, then $\hat{N}=H$ for the topology which is the supremum 
of the topology of uniform convergence on weakly compact subsets of $E, \hat{F}$ having the norm topology and uniform convergence on the unit ball of $E, \hat{F}$ having the $\sigma\left(\hat{F}, F^{\prime}\right)$ topology.

(iv) Let $H$ be all compact operators mapping $E$ into $\hat{F}$. If $F$ has the approximation property then $\hat{N}=H$ for the norm topology.

\section{BIBLIOGRAPHY}

1. J. W. Brace, The topology of almost uniform convergence, Pacific J. Math. 9 (1959), 643-652. MR 22 \#179.

2. - Approximating compact and weakly compact operators, Proc. Amer. Math. Soc. 12 (1961), 392-393. MR 24 \#A435.

3. - Convergence on filters and simple equicontinuity, Illinois J. Math. 9 (1965), 286-296. MR 31 \#710.

4. J. W. Brace and R. M. Nielsen, A uniform boundedness theorem, Proc. Amer. Math. Soc. 18 (1967), 624-627. MR 35 \#3403.

5. J. W. Brace, G. D. Friend and P. J. Richetta, Locally convex topologies on function spaces, Duke Math. J. 36 (1969), 709-714. MR 40 \#6223.

6. N. Dunford and J. T. Schwartz, Linear operators. I: General theory, Pure and Appl. Math., vol. 7, Interscience, New York, 1958. MR 22 \#8302.

7. A. Grothendieck, Produits tensoriels topologiques et espaces nucléaires, Mem. Amer. Math. Soc. No. 16 (1955). MR 17, 763.

8. R. H. Herman, Operator representation theorems, Proc. Amer. Math. Soc. 10 (1968), 372-376. MR 36 \#5755.

9. J. Horváth, Topological vector spaces and distributions. Vol. 1, Addison-Wesley, Reading, Mass., 1966. MR 34 \#4863.

10. J. L. Kelley, General topology, Van Nostrand, Princeton, N. J., 1955. MR 16, 1136.

11. I. Kaplansky, Functional analysis. Some aspects of analysis and probability, Surveys in Appl. Math., vol. 4, Wiley, New York; Chapman \& Hall, London, 1958. MR 21 \#286.

12. I. Singer, On the basis problem in topological linear spaces, Rev. Roumaine Math. Pures Appl. 10 (1965), 453-457. MR 33 \#3081.

13. J. W. Brace, The space of continuous linear operators as a completion of $E^{\prime} \otimes F$, Bull. Amer. Math. Soc. 75 (1969), 821-823. MR 39 \#7393.

UNIVERSITY OF MARYLAND,

College Park, Maryland 20742

LEHIGH UNIVERSITY, Bethlehem, Pennsylvania 18015 